\title{
Optimal Spectrum Sharing for Multi-hop Software Defined Radio Networks
}

\author{
Y. Thomas Hou ${ }^{\dagger} \quad$ Yi Shi ${ }^{\dagger} \quad$ Hanif D. Sherali ${ }^{\ddagger}$ \\ $\dagger$ The Bradley Department of Electrical and Computer Engineering, Virginia Tech, Blacksburg, VA \\ $\ddagger$ The Grado Department of Industrial and Systems Engineering, Virginia Tech, Blacksburg, VA
}

\begin{abstract}
Software Defined Radio (SDR) capitalizes advances in signal processing and radio technology and is capable of reconfiguring RF and switching to desired frequency bands. It is a frequency-agile data communication device that is vastly more powerful than recently proposed multi-channel multiradio (MC-MR) technology. In this paper, we investigate the important problem of multi-hop networking with SDR nodes. For such network, each node has a pool of frequency bands (not necessarily of equal size) that can be used for communication. The uneven size of bands in the radio spectrum prompts the need of further division into sub-bands for optimal spectrum sharing. We characterize behaviors and constraints for such multi-hop SDR network from multiple layers, including modeling of spectrum sharing and sub-band division, scheduling and interference constraints, and flow routing. We give a formal mathematical formulation with the objective of minimizing the required network-wide radio spectrum resource for a set of user sessions. Since such problem formulation falls into mixed integer non-linear programming (MINLP), which is NP-hard in general, we develop a lower bound for the objective by relaxing the integer variables and linearization. Subsequently, we develop a nearoptimal algorithm to this MINLP problem. This algorithm is based on a novel sequential fixing procedure, where the integer variables are determined iteratively via a sequence of linear programming. Simulation results show that solutions obtained by this algorithm are very close to lower bounds obtained via relaxation, thus suggesting that the solution produced by the algorithm is near-optimal.
\end{abstract}

\section{INTRODUCTION}

A Software Defined Radio (SDR) or Cognitive Radio (CR) is a frequency-agile data communication device with a rich control and monitoring (spectrum sensing) interface. ${ }^{1}$ It capitalizes advances in signal processing and radio technology, as well as recent advancements in spectrum policy [16], [20]. A frequency-agile radio module is capable of reconfiguring RF and switching to newly-selected frequency bands. Thus, an SDR can be programmed to tune to and operate on specific frequency bands over a wide range of spectrum [20]. An even more profound advance in SDR technology is that there is no requirement that selected frequencies/channels be contiguous: the radio can send packets over non-contiguous frequency bands. From an application perspective, SDR allows a single

\footnotetext{
${ }^{1} \mathrm{SDR}$ with spectrum sensing and artificial intelligence is also called cognitive radio [8], [16]. In this paper, we use the term SDR to stand for programmable radio in the broad sense (including cognitive radio) when there is no confusion.
}

radio to provide a wide variety of functions, acting as a cell phone, broadcast receiver, GPS receiver, wireless data terminal or node, etc.

It is important to understand that an SDR is vastly more powerful and flexible than recently proposed multi-channel multi-radio (MC-MR) technology (e.g., [1], [2], [6], [10], [12], [13], [11], [17], [19]). First, MC-MR platform employs traditional hardware-based radio technology (i.e., signal processing, modulation etc. are all implemented in hardware) and thus each radio can only operate on a single channel at a time and there is no switching of channel on packet level. As a result, the number of concurrent channels that can be used at a wireless node is limited by the number of hardwarebased radios. In contrast, the radio technology in SDR is software-based; a soft-radio is capable of switching frequency bands on packet level. As a result, the number of concurrent frequency bands that can be shared by a single soft-radio is typically much larger than that can be supported by MC-MR. Second, due to the nature of hardware-based radio technology in MC-MR, a common assumption in MC-MR is that there is a set of "common channels" available for every node in the network; each channel typically has the same bandwidth. However, such assumption is hardly true for SDR networks, in which each node may have a different set of frequency bands, each of un-equal size. Due to this difference, SDR is required to work on a set of "heterogeneous" channels that are scattered on widely-separated slices of the frequency spectrum with different bandwidths. These important differences between MC-MR and SDR warrant that the algorithm design for an SDR network is substantially complex than that under MCMR. In some sense, an MC-MR based wireless network can be considered as a special case of an SDR based wireless network. Thus, algorithms designed for SDR network can be tailored to address MC-MR network while the converse is not true.

In this paper, we focus on the multi-hop networking problem for an SDR-based wireless network. For such network, each node has a set of spectrum bands that it can use. Due to the uneven nature of spectrum bands, it may be necessary to further divide each band into sub-bands (likely uneven) for transmission and reception. There are many fundamental 
problems that can be posed for such wireless networks in the context of rates and capacity. In this paper, we consider the following problem. Suppose there are a set of user sessions in the network that is characterized by a set of sourcedestination pairs each having certain rate requirement. How to perform spectrum allocation, scheduling and interference avoidance, and multi-hop multi-path routing such that the required network-wide radio spectrum resource is minimized?

Our motivation to pursue this problem is two-fold. First, it has been shown in [14] that the so-called space-bandwidth product (SBP) is an extremely useful performance metric in the context of multi-hop SDR network. In the absence of power control, this SBP metric degenerates into the performance objective in this paper. Second, the cross-layer constraints that we will present in this paper is general and characterize common agreed behaviors of packet radio networks. Therefore, the solution procedure can be easily modified to address other performance objectives (e.g., rates or capacity).

To formulate the problem mathematically, we characterize behaviors and constraints from multiple layers for a general multi-hop SDR network. Special attention is given to modeling of spectrum sharing and (un-even) sub-band division, scheduling and interference modeling, and multi-path routing. We formulate an optimization problem with the objective of minimizing the required network-wide radio spectrum resource for a set of source-destination pair rate requirements. Since such problem formulation falls into mixed integer non-linear programming (MINLP), which is NP-hard in general [7], we aim to develop near-optimal solutions.

We develop a near-optimal algorithm to the MINLP problem. As for preparation, we first develop a lower bound for the objective by relaxing the integer variables and linearization. This lower bound can be used as a measure for the quality of any solution. We propose a novel sequential fixing (SF) solution procedure where the determination of integer variables is performed iteratively through a sequence of linear programming (LP). Upon completing of fixing the integer variables, other variables in the optimization problem can be solved with an LP. Since the solution obtained by the proposed $\mathrm{SF}$ algorithm represents an upper bound for the objective, we compare it to the lower bound that we developed earlier. Simulations show that the results obtained by the SF algorithm are very close to the lower bound, thus suggesting that (1) the lower bound is very tight; and (2) the solution obtained by the SF algorithm is even closer to the optimum and thus is near-optimal.

The rest of this paper is organized as follows. In Section II, we review related work on SDR and also discuss current stateof-the-art on cross-layer optimization for MC-MR networks. In Section III, we characterize the behavior of SDR network from multiple layers and formulate them into mathematical constraints. We also elaborate the optimal radio resource sharing problem and formulate it into an MINLP problem. Section IV presents a lower bound for this MINLP problem by relaxing integer variables and linearization. In Section V, we present the SF algorithm. Section VI presents simulation results and demonstrates the near-optimal performance of the SF algorithm. Section VII addresses implementation considerations and Section VIII concludes this paper.

\section{RELATED WORK}

There has been extensive studies on multi-channel multiradio (MC-MR) networks (e.g., [1], [2], [6], [10], [12], [13], [11], [17], [19]). As discussed, SDR is vastly more powerful and flexible than MC-MR. Due to their significant differences in terms of radio hardware technology and how to use available frequency bands, algorithm design for an SDR network is substantially complex than that for MC-MR. An MC-MR based wireless network could be considered as a special case of an SDR based wireless network. We review the following recent work on cross-layer optimization for MC-MR networks and understand the current state-of-the-art on problems for MC-MR networks.

In [2], Alicherry et al. formulated a joint channel assignment and routing problem for an MC-MR network, with the aim of maximizing network throughput (defined as a scaling factor for a given rate vector in the network). The main result is an algorithm that produces a solution within a constant factor of the optimal solution. The constant factor is $K \cdot c(q) / I$ where $K$ is number of channels in the network, $c(q)$ is a constant greater than 4 ( 8 is considered a typical case in the proof in [2]) and $I$ is the number of radios at each node.

In [11], Kodialam and Nandagopal also formulated a crosslayer problem for an MC-MR network, with the aim of maximizing network capacity (also defined as a scaling factor for a given rate vector in the network). The main results are (1) an upper bound for capacity region (through integer relaxation and LP) and (2) a lower bound that is obtained by finding a feasible solution for link channel assignment and scheduling. The feasible solution is obtained by first fixing routes and then finding link channel assignment and scheduling solution, or a layer-decoupling approach. Simulation results show that, for random topology, there is an average of $25 \%$ gap between the feasible solution and upper bound.

Although there is some work on spectrum sharing for single-hop communications (see, e.g., [4], [18] and references therein), there is limited amount of work available in the literature on multi-hop wireless networking with SDRs. In [23], Xin et al. studied how to assign frequency bands at each node to form a topology such that a certain performance metric can be optimized. A layered graph was proposed to model frequency bands available at each node and to facilitate topology formation and achieve optimization objective. The authors considered the so-called fixed channel approach whereby the radio is assumed to operate on only one channel at 
TABLE I

NOTATION.

\begin{tabular}{|c|l|}
\hline Symbol & Definition \\
\hline $\mathcal{N}$ & The set of nodes in the network \\
$\mathcal{L}$ & The set of active user sessions in the network \\
$r(l)$ & Rate of session $l \in \mathcal{L}$ \\
$s(l), d(l)$ & Source and destination nodes of session $l$ \\
$\mathcal{M}_{i}$ & The set of available bands at node $i \in \mathcal{N}$ \\
$\mathcal{M}$ & The set of available bands in the network, i.e., $\bigcup_{i \in \mathcal{N}} \mathcal{M}_{i}$ \\
$M$ & $=|\mathcal{M}|$ \\
$\mathcal{M}_{i j}$ & The set of available bands on link $(i, j)$, i.e., $\mathcal{M}_{i} \cap \mathcal{M}_{j}$ \\
$W^{(m)}$ & Bandwidth of band $m \in \mathcal{M}$ \\
$K^{(m)}$ & Total number of sub-bands in band $m$ \\
$d_{i j}$ & Distance between nodes $i$ and $j$ \\
$n$ & Path loss index \\
$g_{i j}$ & Propagation gain from node $i$ to node $j$ \\
$Q$ & Transmission power spectral density at a transmitter \\
$\eta$ & Ambient Gaussian noise density \\
$Q_{T}$ & The minimum threshold of power spectral density to \\
$Q_{I}$ & decode a transmission at a receiver \\
& The maximum threshold of power spectral density for \\
$R_{T}, R_{I}$ & interference to be negligible at a receiver \\
$\mathcal{T}_{i}^{m}$ & Transmission range and interference range, respectively \\
$\mathcal{T}_{i}$ & The set of nodes that can use band $m$ and are within the \\
$\mathcal{I}_{j}^{m}$ & transmission range to node $i$ \\
$u^{(m, k)}$ & The set of nodes that can use band $m$ and are within the \\
& interference range to node $j$ \\
$x_{i j}^{(m, k)}$ & The fraction of bandwidth for the $k$-th sub-band in \\
$f_{i j}(l)$ & band $m$ \\
& Binary indicator to mark whether or not sub-band $(m, k)$ \\
& is used by link $(i, j)$. \\
Data rate that is attributed to session $l$ on link $(i, j)$
\end{tabular}

a specific time. In [22], Steenstrup studied three different frequency assignment problems: common broadcast frequencies, non-interfering frequencies for simultaneous transmissions, and frequencies for direct source-destination communications. Each is viewed as a graph-coloring problem, and both centralized and distributed algorithms are presented.

\section{SDR Network Model ANd Problem FORMULATION}

Table I lists all notation in this paper. We consider an ad hoc network consisting a set of $\mathcal{N}$ nodes. Among these nodes, there are a set of $\mathcal{L}$ uni-cast communication sessions. Denote $s(l)$ and $d(l)$ the source and destination nodes of session $l \in \mathcal{L}$ and $r(l)$ the rate requirement (in $\mathrm{b} / \mathrm{s}$ ) of session $l$.

\section{A. Modeling of Multi-layer Characteristics}

Modeling of Spectrum Sharing and Sub-band Division. This part of mathematical modeling and constraints are unique to SDR networks and do not exist in MC-MR networks. In a multi-hop SDR network, the available spectrum bands at one node may be different from another node in the network. Given a set of available frequency bands at a node, one additional subtle issue is that the size (or bandwidth) of each band may differ drastically. For example, among the least-utilized spectrum bands found in [15], the bandwidth between $[1240,1300] \mathrm{MHz}$ (allocated to radio amateur) is

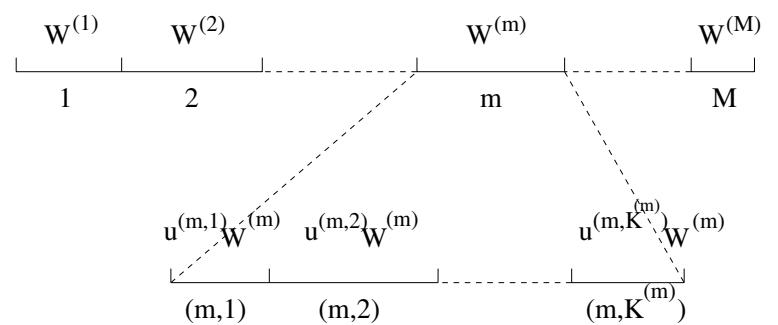

Fig. 1. A schematic illustrating bands and sub-bands concept in spectrum sharing.

$60 \mathrm{MHz}$ while bandwidth between $[1525,1710] \mathrm{MHz}$ (allocated to mobile satellites, GPS systems, and meteorological applications) is $185 \mathrm{MHz}$. Such uneven bandwidth among the available bands suggests the need for further division of the larger bands into smaller sub-bands for more flexible and efficient frequency allocation. Since equal sub-band division of the available spectrum band is likely to yield sub-optimal performance, an un-equal division is necessary.

More formally, we model the union of available spectrum in the network as a set of $M$ un-equal sized bands (see Fig. 1). Denote $\mathcal{M}$ the set of these bands and $\mathcal{M}_{i} \subseteq \mathcal{M}$ the set of available bands (or white space) at node $i \in \mathcal{N}$, which is likely to be different from another node, say $j \in \mathcal{N}$, i.e., $\mathcal{M}_{i} \neq \mathcal{M}_{j}$. For example, at node $i, \mathcal{M}_{i}$ may consist of bands I, III, and $\mathrm{V}$ while at node $j, \mathcal{M}_{j}$ may consist of bands I, IV, and VI. Denote $W^{(m)}$ the bandwidth of band $m \in \mathcal{M}$. To overcome the uneven size of bands in the spectrum, we assume that band $m$ can be further divided into $K^{(m)}$ sub-bands, each of which may be unequal. Denote $u^{(m, k)}$ the fraction of bandwidth for the $k$-th sub-band in band $m$, which is part of our cross-layer optimization variables. Then we have

$$
\sum_{k=1}^{K^{(m)}} u^{(m, k)}=1 .
$$

As an example, Fig. 1 shows $M$ bands in the network and for a specific band $m$, it may be further divided into $K^{(m)}$ sub-bands. Then the $M$ bands in the network are effectively divided into $\sum_{m=1}^{M} K^{(m)}$ sub-bands, each of which may be of different size.

Transmission Range and Interference Range. We assume that the power spectral density from the transmitter of an SDR node is $Q$. In this paper, we assume all nodes use the same power density for transmission. The more complex issue of power control will be deferred for future research. A widelyused model for power propagation gain is

$$
g_{i j}=d_{i j}^{-n},
$$

where $n$ is the path loss index and $d_{i j}$ is the distance between 
nodes $i$ and $j .^{2}$ We assume a data transmission is successful only if the received power spectral density at a receiver exceeds a threshold $Q_{T}$. Likewise, we assume interference will become non-negligible only if it produces a power spectral density over a threshold of $Q_{I}$ at a receiver. Based on the threshold $Q_{T}$, the transmission range for a node is thus $R_{T}=\left(Q / Q_{T}\right)^{1 / n}$, which comes from $g_{i j} \cdot Q=Q_{T}$ and (1). Similarly, based on the interference threshold $Q_{I}\left(<Q_{T}\right)$, the interference range for a node is $R_{I}=\left(Q / Q_{I}\right)^{1 / n}$. Since $Q_{I}<Q_{T}$, we have $R_{I}>R_{T}$. Both transmission range $R_{T}$ and interference range $R_{I}$ will be used in the modeling of interference constraints as follows.

Scheduling and Interference Constraints. Scheduling can be done either in time domain or frequency domain. In this paper, we consider frequency domain sub-band assignment, i.e., how to assign sub-bands at a node for transmission and reception. A feasible scheduling of frequency bands must ensure that there are no interference at the same node and among the nodes.

Suppose that band $m$ is available at both node $i$ and node $j$, i.e., $m \in \mathcal{M}_{i} \cap \mathcal{M}_{j}$. To simplify the notation, let $\mathcal{M}_{i j}=$ $\mathcal{M}_{i} \cap \mathcal{M}_{j}$. Denote

$$
x_{i j}^{(m, k)}= \begin{cases}1 & \begin{array}{l}
\text { If node } i \text { transmits data to node } j \text { on } \\
\text { sub-band }(m, k), \\
0
\end{array} \\
\text { otherwise. }\end{cases}
$$

For a node $i \in \mathcal{N}$ and a band $m \in \mathcal{M}_{i}$, denote $\mathcal{T}_{i}^{m}$ the set of nodes that can use band $m$ and are within the transmission range of node $i$, i.e.,

$$
\mathcal{T}_{i}^{m}=\left\{j: d_{i j} \leq R_{T}, j \neq i, m \in \mathcal{M}_{j}\right\} .
$$

Note that node $i$ cannot transmit to multiple nodes on the same frequency sub-band. We have

$$
\sum_{q \in \mathcal{T}_{i}^{m}} x_{i q}^{(m, k)} \leq 1
$$

Also node $i$ cannot use the same frequency sub-band for transmission and reception, due to "self-interference" at the physical layer. That is, if $x_{i j}^{(m, k)}=1$, then for any $q \in \mathcal{T}_{j}^{m}$, $x_{j q}^{(m, k)}$ must be 0 . In other words, we have

$$
x_{i j}^{(m, k)}+\sum_{q \in \mathcal{T}_{j}^{m}} x_{j q}^{(m, k)} \leq 1 .
$$

Note that in (3), we are referring to a specific node $j$ to which node $i$ is transmitting. If $x_{i j}^{(m, k)}=1$, then $\sum_{q \in \mathcal{T}_{j}^{m}} x_{j q}^{(m, k)}=0$, i.e., node $j$ cannot use the same frequency sub-band $(m, k)$ for transmission. On the other hand, if $x_{i j}^{(m, k)}=0$, then

\footnotetext{
${ }^{2}$ In this paper, we consider a uniform gain model and assume the same gain model on all frequency bands. The case of non-uniform gain model or banddependent gain behavior can be extended without much technical difficulty.
}

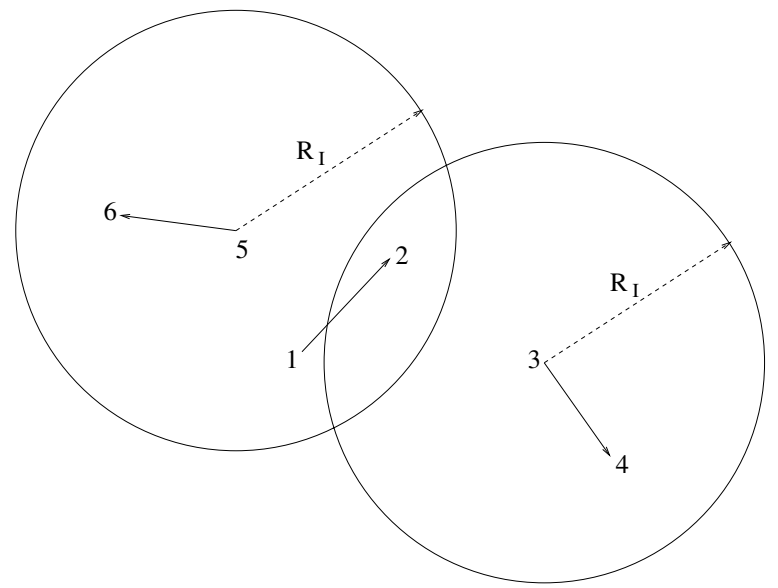

Fig. 2. An example illustrating interference among links.

$\sum_{q \in \mathcal{T}_{j}^{m}} x_{j q}^{(m, k)} \leq 1$, i.e., node $j$ may use frequency sub-band $(m, k)$ for transmission, but can only use it for one receiving node $q \in \mathcal{T}_{j}^{m}$ (same as in (2)).

In addition to the above constraints at the same node, there are also constraints on frequency sub-band use due to potential interference among the nodes in the network. In particular, for a frequency sub-band $(m, k)$, if node $i$ uses this sub-band for transmitting data to a node $j \in \mathcal{T}_{i}{ }^{m}$, then any other node that can produce interference on node $j$ should not use this sub-band. ${ }^{3}$ To model this constraint, we denote $\mathcal{P}_{j}^{m}$ the set of nodes that can produce interference on node $j$ on band $m$, i.e.,

$$
\mathcal{P}_{j}^{m}=\left\{p: d_{p j} \leq R_{I}, p \neq j, \mathcal{T}_{p}^{m} \neq \emptyset\right\} .
$$

The physical meaning of $\mathcal{T}_{p}^{m} \neq \emptyset$ in the above definition for $\mathcal{P}_{j}^{m}$ is that the interference node $p$ can use band $m$ for a valid transmission to a node in $\mathcal{T}_{p}^{m}$. Then we have

$$
x_{i j}^{(m, k)}+\sum_{q \in \mathcal{T}_{p}^{m}} x_{p q}^{(m, k)} \leq 1 \quad\left(p \in \mathcal{P}_{j}^{m}, p \neq i\right) .
$$

In (4), if $x_{i j}^{(m, k)}=1$, i.e., node $i$ using frequency sub-band $(m, k)$ to transmit to node $j$, then any node $p$ that can produce interference on node $j$ should not transmit on this sub-band, i.e., $\sum_{q \in \mathcal{T}_{p}^{m}} x_{p q}^{(m, k)}=0$. On the other hand, if $x_{i j}^{(m, k)}=0$, (4) degenerates into (2), i.e., node $p$ may transmit on sub-band $(m, k)$ to one node $q \in \mathcal{T}_{p}^{m}$, i.e., $\sum_{q \in \mathcal{T}_{p}^{m}} x_{p q}^{(m, k)} \leq 1$.

It is important to understand that in the interference constraint (4), if $x_{i j}^{(m, k)}=0$, two nodes that can produce interference on node $j$ but are far apart and outside each other's interference range can use the same sub-band $(m, k)$ for transmission. We use an example to illustrate this point. In Fig. 2, suppose node 1 is transmitting to node 2 on subband $(m, k)$, then any node that can produce interference on

\footnotetext{
${ }^{3}$ Note that the so-called "hidden terminal" problem is a special case under this constraint.
} 
node 2 (i.e., node 3 or 5 ) cannot use the same sub-band for transmission. On the other hand, if node 1 is not using subband $(m, k)$ to transmit to node 2 , then node 3 may use this sub-band to transmit (to node 4) as stated in (4). Likewise, node 5 may also use this sub-band to transmit (to node 6 ) as stated in (4). That is, both nodes 3 and 5 may use the same sub-band for transmission.

Routing. At the network level, a source node may need a number of relay nodes to route the data stream toward its destination node. Clearly, a route having a single path may be overly restrictive and is not able to take advantage of load balancing. A set of paths (or multi-path) is more flexible to route the traffic from a source node to its destination. Mathematically, this can be modeled as follows.

Denote $f_{i j}(l)$ the data rate on link $(i, j)$ that is attributed to session $l$, where $i \in \mathcal{N}, j \in \bigcup_{m \in \mathcal{M}_{i}} \mathcal{T}_{i}^{m}$, and $l \in \mathcal{L}$. To simplify the notation, let $\mathcal{T}_{i}=\bigcup_{m \in \mathcal{M}_{i}} \mathcal{T}_{i}^{m}$. If node $i$ is the source node of session $l$, i.e., $i=s(l)$, then

$$
\sum_{j \in \mathcal{T}_{i}} f_{i j}(l)=r(l) \text {. }
$$

If node $i$ is an intermediate relay node for session $l$, i.e., $i \neq$ $s(l)$ and $i \neq d(l)$, then

$$
\sum_{j \in \mathcal{T}_{i}}^{j \neq s(l)} f_{i j}(l)=\sum_{p \in \mathcal{T}_{i}}^{p \neq d(l)} f_{p i}(l)
$$

If node $i$ is the destination node of session $l$, i.e., $i=d(l)$, then

$$
\sum_{p \in \mathcal{T}_{i}} f_{p i}(l)=r(l) .
$$

It can be easily verified that if (5) and (6) are satisfied, (7) must be satisfied. As a result, it is sufficient to list (5) and (6) in the formulation.

In addition to the above flow balance equations at each node $i$ for each session $l$, the aggregate flow rates on each radio link cannot exceed this link's capacity. To model this mathematically, we need to first find the capacity on link $(i, j)$ in sub-band $(m, k)$. If node $i$ sends data to node $j$ on subband $(m, k)$, i.e., $x_{i j}^{(m, k)}=1$, then the capacity on link $(i, j)$ in sub-band $(m, k)$ is

$$
c_{i j}^{(m, k)}=u^{(m, k)} W^{(m)} \log _{2}\left(1+\frac{g_{i j} Q}{\eta}\right),
$$

where $\eta$ is the ambient Gaussian noise density. Note that the denominator inside the $\log$ function contains only $\eta$. This is due to one of our interference constraints stated earlier, i.e., when node $i$ is transmitting to node $j$ on sub-band $(m, k)$, then all the other neighbors of node $j$ within its interference range are refrained from using this sub-band. This interference constraint significantly helps to simplify the calculation of link capacity $c_{i j}^{(m, k)}$. When $x_{i j}^{(m, k)}=0$, we have $c_{i j}^{(m, k)}=0$. Thus, $c_{i j}^{(m, k)}$ can be written in the following compact form.

$$
c_{i j}^{(m, k)}=x_{i j}^{(m, k)} \cdot u^{(m, k)} W^{(m)} \log _{2}\left(1+\frac{g_{i j} Q}{\eta}\right) .
$$

Now getting back to our earlier requirement that the aggregate data rates on each link $(i, j)$ cannot exceed the link's capacity, we have

$$
\begin{aligned}
& \sum_{l \in \mathcal{L}}^{s(l) \neq j, d(l) \neq i} f_{i j}(l) \leq \sum_{m \in \mathcal{M}_{i j}} \sum_{k=1}^{K^{(m)}} c_{i j}^{(m, k)} \\
= & \sum_{m \in \mathcal{M}_{i j}} \sum_{k=1}^{K^{(m)}} x_{i j}^{(m, k)} \cdot u^{(m, k)} W^{(m)} \log _{2}\left(1+\frac{g_{i j} Q}{\eta}\right) .
\end{aligned}
$$

\section{B. Problem Formulation}

For the multi-hop SDR networks we are investigating, various performance objectives can be used as optimization objective (e.g., network throughput as measured by a scaling factor of rates of flows in the network [2], [11]). In this paper, we use the total required radio resource to support the user sessions as our performance objective. Such radio resource can be measured in terms of the total bandwidth used by all nodes in the network, which is the simplified form of the socalled space-bandwidth product (SBP) proposed in [14] when transmission power spectral density is fixed. It is not hard to see that the solution procedure in this paper is not constrained by the particular objective. The solution procedure can be easily modified to tailor other performance objectives if needed (e.g., rates or capacity).

To re-cap, we are given a set of source-destination pairs (user sessions) in the network each with a certain rate requirement. Each node in the network has a set of available frequency bands that it can use for communication. We want to find the optimal solution to divide the set of available frequency bands at each node, the scheduling of sub-bands for transmission and reception, and multi-hop routing for each flow such that the total radio bandwidth used in the network is minimized (or the solution declares that there is no feasible solution). Mathematically, we have the following optimization problem.

$\operatorname{Min} \sum_{i \in \mathcal{N}} \sum_{m \in \mathcal{M}_{i}} \sum_{j \in \mathcal{T}_{i}^{m}} \sum_{k=1}^{K^{(m)}} W^{(m)} x_{i j}^{(m, k)} u^{(m, k)}$

$$
\begin{array}{cc}
\text { s.t. } \quad \sum_{k=1}^{K^{(m)}} u^{(m, k)}=1 & (m \in \mathcal{M}) \\
\sum_{q \in \mathcal{T}_{i}^{m}} x_{i q}^{(m, k)} \leq 1 & \left(i \in \mathcal{N}, m \in \mathcal{M}_{i}, 1 \leq k \leq K^{(m)}\right)(9) \\
x_{i j}^{(m, k)}+\sum_{q \in \mathcal{T}_{p}^{m}} x_{p q}^{(m, k)} \leq 1 & \left(i \in \mathcal{N}, m \in \mathcal{M}_{i}, j \in \mathcal{T}_{i}^{m},\right. \\
& \left.1 \leq k \leq K^{(m)}, p \in \mathcal{I}_{j}^{m}, p \neq i\right)(10)
\end{array}
$$




$$
\begin{aligned}
& \sum_{l \in \mathcal{L}}^{s(l) \neq j, d(l) \neq i} f_{i j}(l)-\sum_{m \in \mathcal{M}_{i j}} \sum_{k=1}^{K^{(m)}} W^{(m)} \log _{2}\left(1+\frac{g_{i j} Q}{\eta}\right) x_{i j}^{(m, k)} u^{(m, k)} \\
& \leq 0 \\
& \quad\left(i \in \mathcal{N}, j \in \mathcal{T}_{i}\right)
\end{aligned}
$$

$$
\begin{array}{rc}
\sum_{j \in \mathcal{T}_{i}} f_{i j}(l)=r(l) & (l \in \mathcal{L}, i=s(l)) \\
\sum_{j \in \mathcal{T}_{i}}^{j \neq s(l)} f_{i j}(l)-\sum_{p \in \mathcal{T}_{i}}^{p \neq d(l)} f_{p i}(l) & =0 \quad(l \in \mathcal{L}, i \in \mathcal{N}, i \neq s(l), d(l)) \\
x_{i j}^{(m, k)}=0 \text { or } 1, u^{(m, k)} \geq 0 & \left(i \in \mathcal{N}, m \in \mathcal{M}_{i}, j \in \mathcal{T}_{i}^{m}, 1 \leq k \leq K^{(m)}\right) \\
f_{i j}(l) & \geq 0 \quad\left(l \in \mathcal{L}, i \in \mathcal{N}, i \neq d(l), j \in \mathcal{T}_{i}, j \neq s(l)\right),
\end{array}
$$

$$
\operatorname{Min} \sum_{i \in \mathcal{N}} \sum_{m \in \mathcal{M}_{i}} \sum_{j \in \mathcal{T}_{i}^{m}} \sum_{k=1}^{K^{(m)}} W^{(m)} s_{i j}^{(m, k)}
$$

$$
\begin{array}{lcc}
\text { s.t. } \quad \sum_{k=1}^{K^{(m)}} u^{(m, k)}=1 & (m \in \mathcal{M}) \\
\sum_{q \in \mathcal{T}_{i}^{m}} s_{i q}^{(m, k)}-u^{(m, k)} \leq 0 & \left(i \in \mathcal{N}, m \in \mathcal{M}_{i}, 1 \leq k \leq K^{(m)}\right)(11) \\
s_{i j}^{(m, k)}+\sum_{q \in \mathcal{T}_{p}^{m}} s_{p q}^{(m, k)}-u^{(m, k)} \leq 0 & \left(i \in \mathcal{N}, m \in \mathcal{M}_{i}, j \in \mathcal{T}_{i}^{m},\right. \\
& \left.1 \leq k \leq K^{(m)}, p \in \mathcal{I}_{j}^{m}, p \neq i\right)(12)
\end{array}
$$

where $W^{(m)}, g_{i j}, Q, \eta$, and $r(l)$ are all constants and $x_{i j}^{(m, k)}$,s, $u^{(m, k)}$ 's, and $f_{i j}(l)$ 's are all optimization variables. In (10), $\mathcal{I}_{j}^{m}$ is defined as

$$
\mathcal{I}_{j}^{m}=\left\{p: d_{p j} \leq R_{I}, \mathcal{T}_{p}^{m} \neq \emptyset\right\}
$$

which is equivalent to

$$
\mathcal{I}_{j}^{m}= \begin{cases}\mathcal{P}_{j}^{m} \bigcup\{j\} & \text { If } \mathcal{T}_{j}^{m} \neq \emptyset \\ \mathcal{P}_{j}^{m} & \text { otherwise } .\end{cases}
$$

This compact form enables (10) to include both (3) and (4) in the formulation.

The above optimization problem is in the form of mixedinteger non-linear programming (MINLP) problem, which is NP-hard in general [7]. Although existing software (e.g. BARON [3]) could solve very small-sized network (e.g., several nodes), the time complexity becomes prohibitively high when it comes to larger networks.

Our approach to this problem is as follows. In Section IV, we first explore a lower bound for the objective, which can be obtained by relaxing the integer variables and linearization. Using this lower bound as a performance benchmark, in Section V, we develop a highly effective algorithm based on a novel sequential fixing (SF) procedure. Using simulation results, we show that the SF algorithm has a performance very close to the lower bound. Since the optimal objective value lies between the lower bound and the solution obtained by the SF algorithm, the heuristic solution must be even closer to the true optimum.

\section{A LOWER BOUND FOR THE OBJECTIVE FunCtion}

The complexity of the problem formulated in Section IIIB arises from the integer variables $x_{i j}^{(m, k)}$,s and product of variables $x_{i j}^{(m, k)} u^{(m, k)}$ 's. To pursue a lower bound for the objective, we can relax the integer (binary) requirement on $x_{i j}^{(m, k)}$ with $0 \leq x_{i j}^{(m, k)} \leq 1$ and replace $x_{i j}^{(m, k)} u^{(m, k)}$ with a single variable, say $s_{i j}^{(m, k)}$, i.e., $s_{i j}^{(m, k)}=x_{i j}^{(m, k)} u^{(m, k)} \leq$ $u^{(m, k)}$. Such relaxation enables us to have the following problem formulation.

$$
\begin{array}{r}
\sum_{l \in \mathcal{L}}^{s(l) \neq j, d(l) \neq i} f_{i j}(l)-\sum_{m \in \mathcal{M}_{i j}} \sum_{k=1}^{K^{(m)}} W^{(m)} \log _{2}\left(1+\frac{g_{i j} Q}{\eta}\right) s_{i j}^{(m, k)} \leq 0 \\
\left(i \in \mathcal{N}, j \in \mathcal{T}_{i}\right)
\end{array}
$$

$$
\begin{gathered}
\sum_{j \in \mathcal{T}_{i}} f_{i j}(l)=r(l) \quad(l \in \mathcal{L}, i=s(l)) \\
\sum_{j \in \mathcal{T}_{i}}^{j \neq s(l)} f_{i j}(l)-\sum_{p \in \mathcal{T}_{i}}^{p \neq d(l)} f_{p i}(l)=0 \quad(l \in \mathcal{L}, i \in \mathcal{N}, i \neq s(l), d(l)) \\
u^{(m, k)}, s_{i j}^{(m, k)} \geq 0 \quad\left(i \in \mathcal{N}, m \in \mathcal{M}_{i}, j \in \mathcal{T}_{i}^{m}, 1 \leq k \leq K^{(m)}\right) \\
f_{i j}(l) \geq 0 \quad\left(l \in \mathcal{L}, i \in \mathcal{N}, i \neq d(l), j \in \mathcal{T}_{i}, j \neq s(l)\right)
\end{gathered}
$$

This new (relaxed) formulation falls into a standard linear programming (LP) problem, the solution of which can be obtained in polynomial time. Due to the relaxation (and thus enlarged optimization space), the solution to this LP problem corresponds to a lower bound to the objective of the original problem in Section III-B. There may not exist a feasible solution to achieve this lower bound.

Nevertheless, this lower bound offers a benchmark to measure the quality of a feasible solution, which we will develop in the next section. It turns out that this lower bound is extremely tight (see results in Section V). This can be explained by the the convex hull results presented by Sherali et al. in [21].

\section{A Near-Optimal Algorithm Based on SEQUential FiXing}

\section{A. Basic Algorithm}

We now take a closer look at the original MINLP problem formulation in Section III-B. Observe that once the binary values for $x_{i j}^{(m, k)}$ are determined, i.e., whether or not a node will indeed use certain sub-band to send data to another node, then this MINLP reduces to an LP, which can be solved in polynomial time. In other words, the key obstacle in solving this MINLP problem lies in the determination of the binary values for $x_{i j}^{(m, k)}$,s. To this end, we propose a twostep solution procedure: i) fix the binary values for $x_{i j}^{(m, k)}$ 
Sequential Fixing (SF) Algorithm

1. Set up and solve the initial relaxed LP problem as shown in Section IV.

2. Suppose $x_{i j}^{(m, k)}$ is the largest among all the $x$ values that remain to be fixed, fix this $x_{i j}^{(m, k)}=1$.

Also fix $x_{i q}^{(m, k)}=0$ (for $q \in \mathcal{T}_{i}^{m}$ and $q \neq j$ ) and

$x_{p q}^{(m, k)}=0$ (for $p \in \mathcal{I}_{j}^{m}, p \neq i$, and $q \in \mathcal{T}_{p}^{m}$ ).

3. If all $x_{i j}^{(m, k)}$ are fixed, go to Step 5 .

4. Otherwise, reformulate and solve a new relaxed LP problem with newly fixed $x$ variables and go to Step 2 .

5. Formulate an LP problem based on all fixed $x$ values. Obtain a solution to this LP problem.

Fig. 3. Sequential Fixing (SF) algorithm.

iteratively through a sequence of LPs; ii) once all $x_{i j}^{(m, k)}$,s are fixed, find a solution (consisting of how to divide sub-bands and flow routing) corresponding to this set $x_{i j}^{(m, k)}$. Such twostep approach will yield a sub-optimal (upper bound) solution to the original MINLP problem. The quality of this algorithm can be assessed by how close its solution is to the lower bound we developed in the last section.

As said, the key to the two-step approach resides in the determination of the binary values for all $x_{i j}^{(m, k)}$, s. Our main idea is to fix (set) the values of $x_{i j}^{(m, k)}$,s sequentially through solving a series of relaxed LP problems, with each iteration to set at least one binary value for some $x_{i j}^{(m, k)}$,s. Specifically, during the first iteration, we relax all binary variables $x_{i j}^{(m, k)}$ to $0 \leq x_{i j}^{(m, k)} \leq 1$ as in Section IV to have an LP. Upon solving this LP, we have a solution with each $x_{i j}^{(m, k)}=s_{i j}^{(m, k)} / u^{(m, k)}$ being a fraction between 0 and 1 . Among all the $x$ fractions, suppose some $x_{i j}^{(m, k)}$ has the largest value. Then we fix (set) this particular $x_{i j}^{(m, k)}$ to 1 . As a result of this fixing, by (9), we also need to fix $x_{i q}^{(m, k)}=0$ for $q \in \mathcal{T}_{i}^{m}$ and $q \neq j$. By (10), we also fix $x_{p q}^{(m, k)}$ to 0 for $p \in \mathcal{I}_{j}^{m}, p \neq i$, and $q \in \mathcal{T}_{p}^{m}$.

Now we have fixed some $x$ variables in the first iteration. We update a new LP for the second iteration as follows. For those $x_{i j}^{(m, k)}$,s that are already fixed as 1 , since $s_{i j}^{(m, k)}=$ $x_{i j}^{(m, k)} u^{(m, k)}=u^{(m, k)}$, we can replace the corresponding $s_{i j}^{(m, k)}$ 's by $u^{(m, k)}$ 's. For those $x_{i q}^{(m, k)}$ 's and $x_{p q}^{(m, k)}$, s that are fixed to 0 , we can set $s_{i q}^{(m, k)}=0$ and $s_{p q}^{(m, k)}=0$. As a result, all the terms in the LP involving these $s$ variables can be removed and the corresponding constraint in (11) and (12) can also be removed.

In the second iteration, we can solve this new LP and then fix some additional $x$ variables based on the same process (now the ordering of $x$ 's values is done only for the remaining un-fixed $x$ variables). The iteration continues and eventually we can determine all $x$ variables to either 0 and 1 .
TABLE II

AVAILABLE BANDS AMONG ALL NODES IN THE NETWORK IN THE SIMULATION STUDY.

\begin{tabular}{|c|c|c|}
\hline Band Index & Spectrum Range $(\mathrm{MHz})$ & Bandwidth $(\mathrm{MHz})$ \\
\hline I & {$[1240,1300]$} & 60.0 \\
\hline II & {$[1525,1710]$} & 185.0 \\
\hline III & {$[902,928]$} & 26.0 \\
\hline IV & {$[2400,2483.5]$} & 83.5 \\
\hline V & {$[5725,5850]$} & 125.0 \\
\hline
\end{tabular}

Upon fixing all the $x$ values, the original MINLP reduces to an LP problem, which can be solved in polynomial time. Unlike the solutions obtained in Section IV, the final solution obtained here is a feasible solution since all $x$ values are binary instead of fractions. The complete Sequential Fixing (SF) algorithm is given in Fig. 3.

\section{B. An Iteration-Speedup Technique}

In SF algorithm, we need to solve a sequence of LPs. The complexity of SF is polynomial. By exploiting the space and frequency dimensions involved in radio resource allocation, we may decrease the number of LPs by fixing more $x$ variables during each iteration in Fig. 3. As a result, the complexity can be further decreased. From space dimension, a sub-band usage will only have an impact within the interference range and the same sub-band can be used by other links outside this range. Thus, for the same sub-band $(m, k)$, we can then fix multiple links that have non-overlapping interference range within one iteration of the sequential fixing algorithm. From frequency dimension, the transmission in one sub-band will not interfere transmission in a different sub-band. Thus, for the same link $(i, j)$, we can then fix multiple sub-bands within one iteration of the SF algorithm. Specifically, we can use a threshold $\alpha>$ 0.5 in this fixing process and fix all $x$ variables exceeding $\alpha$ to 1 in one iteration. Note that $\alpha>0.5$ ensures that both constraints (9) and (10) (interference constraints at each node and among the nodes) will hold during the SF procedure. ${ }^{4}$ In the case that none of the $x$ variable exceeds $\alpha$, we will fall back to the basic algorithm in Fig. 3 and simply choose the largest $x$.

\section{Simulation Results}

In this section, we present simulation results for our SF algorithm and compare it to the lower bound obtained in Section IV. The units for distance, rate, and power density satisfy (1) and (8) and are all normalized with appropriate dimensions. We consider $|\mathcal{N}|=20$ nodes in a $50 \times 50$ area. ${ }^{5}$ Among these 20 nodes, there are $|\mathcal{L}|=5$ active sessions, each with a rate within $[10,100]$.

\footnotetext{
${ }^{4}$ We use $\alpha=0.85$ in our simulation results.

${ }^{5}$ Additional simulation results for 30 -node and 40-node networks can be found in [9].
} 


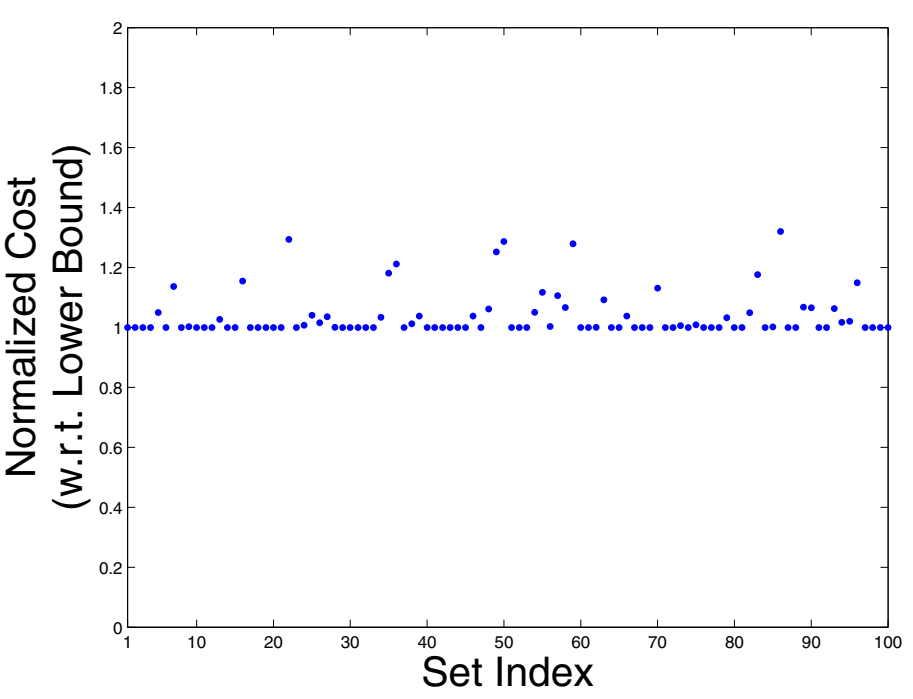

Fig. 4. Normalized cost (with respect to lower bound) for 100 data sets.

We assume there are $M=5$ bands that can be used for the entire network (see Table II). Bands I and II are among the least-utilized (less than 2\%) spectrum bands found in [15] and bands III, IV, and V are unlicensed ISM bands used for 802.11. Recall that available bands at each SDR node is a subset of these 5 bands based on its location and the available bands at any two nodes in the network may not be identical. In the simulation, this is done by randomly selecting a subset of bands from the pool of 5 bands for each node. Further, we assume bands I to $\mathrm{V}$ can be divided into $3,5,2,4$, and 4 sub-bands although other desirable divisions can be used. Note that the size of each sub-band may be un-equal and is part of the optimization problem.

We assume the transmission range on each node is 20 and the interference range is 30 , although other setting can be used. The pass loss index $n$ is assumed to be 4 . The threshold $Q_{T}$ is assumed to be $\eta$. Thus, we have $Q_{I}=\left(\frac{20}{30}\right)^{n} Q_{T}=\frac{16}{81} \eta$ and the transmission power spectral density $Q=(20)^{n} Q_{T}=$ $1.6 \cdot 10^{5} \eta$.

Note that it is possible that for a specific data set, there is no feasible solution. This could be attributed to dis-connectivity in the network (due to random network topology), resource bottleneck in a hot area, etc. In such scenario, there is no numerical result that can be shown for comparison. Thus, we will only show results based on those data sets that have feasible solutions.

We show simulation results for 100 data sets that can produce feasible solutions. For each data set, the network topology, source/destination pair and bit rate of each session, and available frequency bands at each node are randomly generated. We use the SF Algorithm to determine the cost, which is total required bandwidth in the objective function. As discussed, we compare this result with the lower bound developed in Section IV. The running time for each simulation
TABLE III

SIMULATION RESULTS FOR THE FIRST 40 DATA SETS.

\begin{tabular}{|c|c|c||c|c|c|}
\hline $\begin{array}{c}\text { Data Set } \\
\text { Index }\end{array}$ & $\begin{array}{c}\text { Lower } \\
\text { Bound }\end{array}$ & $\begin{array}{c}\text { Result } \\
\text { by SF }\end{array}$ & $\begin{array}{c}\text { Data Set } \\
\text { Index }\end{array}$ & $\begin{array}{c}\text { Lower } \\
\text { Bound }\end{array}$ & $\begin{array}{c}\text { Result } \\
\text { by SF }\end{array}$ \\
\hline 1 & 138.33 & 138.33 & 21 & 156.43 & 156.43 \\
\hline 2 & 156.12 & 156.12 & 22 & 238.41 & 308.51 \\
\hline 3 & 173.53 & 173.53 & 23 & 184.78 & 184.78 \\
\hline 4 & 189.70 & 189.70 & 24 & 241.42 & 243.22 \\
\hline 5 & 203.05 & 213.18 & 25 & 135.39 & 140.96 \\
\hline 6 & 184.37 & 184.37 & 26 & 247.30 & 251.18 \\
\hline 7 & 160.45 & 182.33 & 27 & 280.80 & 290.85 \\
\hline 8 & 232.23 & 232.23 & 28 & 353.98 & 354.17 \\
\hline 9 & 223.00 & 223.53 & 29 & 260.56 & 260.56 \\
\hline 10 & 182.13 & 182.13 & 30 & 127.06 & 127.06 \\
\hline 11 & 220.20 & 220.20 & 31 & 170.35 & 170.35 \\
\hline 12 & 277.83 & 277.83 & 32 & 207.74 & 207.74 \\
\hline 13 & 130.54 & 134.05 & 33 & 183.59 & 183.59 \\
\hline 14 & 172.62 & 172.62 & 34 & 138.33 & 143.00 \\
\hline 15 & 256.96 & 256.96 & 35 & 270.76 & 319.71 \\
\hline 16 & 178.73 & 178.73 & 36 & 325.59 & 394.43 \\
\hline 17 & 152.08 & 152.08 & 37 & 288.72 & 288.72 \\
\hline 18 & 359.03 & 359.03 & 38 & 244.77 & 247.74 \\
\hline 19 & 150.61 & 150.61 & 39 & 215.72 & 223.83 \\
\hline 20 & 164.97 & 164.97 & 40 & 126.05 & 126.05 \\
\hline
\end{tabular}

is less than 10 seconds on a Pentium $3.4 \mathrm{GHz}$ machine.

Figure 4 shows the results of normalized cost obtained by the SF algorithm with respect to the lower bound cost for 100 data sets. The average normalized cost among the 100 simulations is 1.04 and the standard derivation is 0.07 . There are two observations that can be made from this figure. First, since the ratio of the solution obtained by SF (upper bound of optimal solution) to the lower bound solution is close to 1 (in many cases, they coincide with each other), the gap between them is very narrow. Second, since the optimal solution (unknown) is between the solution obtained by the $\mathrm{SF}$ algorithm and the lower bound, the lower bound is very tight; more important, the SF solution must be even closer to the optimum.

To get a sense of how the actual (rather than normalized) numerical results look like in the simulations, we list the first 40 set of results in Table III. Note that in many cases, the result obtained by the SF algorithm is identical to the respective lower bound obtained via relaxation. This indicates that the result found by SF is optimal.

\section{IMPLEMENTATION CONSIDERATIONS}

The SF algorithm as it stands is a centralized algorithm. But this does not prohibit it from being implemented in the realworld for practical applications, just like Dijkstra's algorithm and OLSR [5] are both centralized (link state) and are used for the Internet (OSPF) and ad hoc network, respectively.

We describe two scenarios that the SF algorithm can be implemented in practice. The first scenario, as expected, is based on link state paradigm. In this setting, we could use a common control channel for all the nodes in the network to exchange local state information. Upon such information is 
propagated and shared by all the nodes in the network, each source node can invoke its SF algorithm and compute its route from source to destination. Source routing could be used for packet forwarding to reduce the route computation on nonsource nodes.

In the second scenario, we could use a pre-designated node as a "server node" to which all the other nodes in the network send their local state information. This server node could then compute routes and deliver routing tables to all the nodes in the network. Apparently, "single-point-of-failure" is a problem in this implementation scenario and thus it is necessary to have additional backup server nodes in the network to add redundancies.

\section{CONCLUSIONS}

In this paper, we conducted a systematic study on the important problem of multi-hop networking with SDR nodes. The nature of the problem calls for characterization and modeling of multi-layer behaviors and constraints. We characterized behaviors and constraints for a multi-hop SDR network from multiple layers, including modeling of spectrum sharing and sub-band division, scheduling and interference constraints, and flow routing. We formulated an optimization problem with the objective of minimizing the required network-wide radio spectrum resource for a set of user sessions. Since the problem formulation is an MINLP, we developed a lower bound to estimate the objective function. Subsequently, we developed a novel sequential fixing algorithm to the crosslayer optimization problem. Simulation results showed that solutions obtained by this algorithm are very close to the lower bound, thus suggesting that they are near-optimal.

\section{ACKNOWLEDGEMENTS}

The work of Y.T. Hou and Y. Shi has been supported in part by NSF Grant CNS-0627436. The work of H.D. Sherali has been supported in part by NSF under Grant 0552676 .

\section{REFERENCES}

[1] A. Adya, P. Bahl, J. Padhye, A. Wolman, and L. Zhou, "A multi-radio unification protocol for IEEE 802.11 wireless networks," in Proc. IEEE International Conference on Broadband Networks, pp. 344-354, San Jose, CA, Oct. 25-29, 2004.

[2] M. Alicherry, R. Bhatia, and L. Li, "Joint channel assignment and routing for throughput optimization in multi-radio wireless mesh networks," in Proc. ACM Mobicom, pp. 58-72, August 2005, Cologne, Germany.

[3] BARON Global Optimization Software, http://archimedes.scs.uiuc.edu/ baron/baron.html.

[4] L. Cao and H. Zheng, "Distributed spectrum allocation via local bargaining," in Proc. IEEE Communications Society Conference on Sensor, Mesh, and Ad Hoc Communications and Networks, pp. 475-486, Santa Clara, CA, Sep. 26-29, 2005.

[5] T. Clausen, P. Jacquet, A. Laouiti, P. Muhlethaler, A. Qayyum, and L. Viennot, "Optimized link state routing protocol (OLSR)," RFC 3626, Internet Engineering Task Force, Oct. 2003
[6] R. Draves, J. Padhye, and B. Zill, "Routing in multi-radio, multihop wireless mesh networks," in Proc. ACM Mobicom, pp. 114-128, Philadelphia, PA, Sep. 26-Oct. 1, 2004.

[7] M.R. Garey and D.S. Johnson, Computers and Intractability: A Guide to the Theory of NP-completeness, W.H. Freeman and Company, pp. 245248, New York, NY, 1979.

[8] S. Haykin, "Cognitive radio: Brain-empowered wireless communications," IEEE Journal on Selected Areas in Communications, vol. 23 , no. 2, pp. 201-220, Feb. 2005.

[9] Y.T. Hou, Y. Shi, and H.D. Sherali, "Optimal spectrum sharing for multihop software defined radio networks," Technical Report, the Bradley Department of Electrical and Computer Engineering, Virginia Tech, Blacksburg, VA, July 2006. Available at http://www.ece.vt.edu/thou/ Research.html.

[10] N. Jain, S.R. Das, and A. Nasipuri, "A multichannel CSMA MAC protocol with receiver-based channel selection for multihop wireless networks," in Proc. IEEE International Conference on Computer Communication and Networks, pp. 432-439, Scottsdale, AZ, Oct. 15-17, 2001.

[11] M. Kodialam and T. Nandagopal, "Characterizing the capacity region in multi-radio multi-channel wireless mesh networks," in Proc. ACM Mobicom, pp. 73-87, Cologne, Germany, Aug. 28-Sep. 2, 2005.

[12] P. Kyasanur and N.H. Vaidya, "Routing and interface assignment in multi-channel multi-interface wireless networks," in Proc. IEEE Wireless Communications and Networking Conference, pp. 2051-2056, New Orleans, LA, March 13-17, 2005.

[13] P. Kyasanur and N.H. Vaidya, "Capacity of multi-channel wireless networks: impact of number of channels and interfaces," in Proc. IEEE Mobicom, pp. 43-57, Cologne, Germany, Aug. 28-Sep. 2, 2005.

[14] X. Liu and W. Wang, "On the characteristics of spectrum-agile communication networks," in Proc. IEEE Symposium on New Frontiers in Dynamic Spectrum Access Networks, pp. 214-223, Baltimore, MD, Nov. 8-11, 2005.

[15] M. McHenry and D. McCloskey, "New York City Spectrum Occupancy Measurements September 2004," available at http://www. sharedspectrum.com/inc/content/measurements/nsf/NYC_report.pdf.

[16] J. Mitola III, Cognitive Radio: An Integrated Agent Architecture for Software Defined Radio, Ph.D. thesis, KTH Royal Institute of Technology, 2000.

[17] A. Nasipuri, J. Zhuang, and S.R. Das, "A multichannel CSMA MAC protocol for multihop wireless networks," in Proc. IEEE Wireless Communications and Networking Conference, pp. 1402-1406, New Orleans, LA, Sep. 21-24, 1999.

[18] C. Peng, H. Zheng, B.Y. Zhao, "Utilization and fairness in spectrum assignment for opportunistic spectrum access," ACM/Springer MONET, vol. 11, issue 4, pp. 555-576, Aug. 2006.

[19] A. Raniwala and T. Chiueh, "Architecture and algorithms for an IEEE 802.11-based multi-channel wireless mesh network," in Proc. IEEE Infocom, pp. 2223-2234, Miami, FL, March 13-17, 2005.

[20] J.H. Reed, Software Radio: A Modern Approach to Radio Engineering, Prentice Hall, May 2002.

[21] H.D. Sherali, W.P. Adams, and P.J. Driscoll, "Exploiting special structures in constructing a hierarchy of relaxations for 0-1 mixed integer problems," Operations Research, vol. 46, no. 3, pp. 396-405, 1998.

[22] M.E. Steenstrup, "Opportunistic use of radio-frequency spectrum: A network perspective," in Proc. IEEE Symposium on New Frontiers in Dynamic Spectrum Access Networks, pp. 638-641, Baltimore, MD, Nov. 8-11, 2005.

[23] C. Xin, B. Xie, and C.-C. Shen, "A novel layered graph model for topology formation and routing in dynamic spectrum access networks," in Proc. IEEE Symposium on New Frontiers in Dynamic Spectrum Access Networks, pp. 308-317, Baltimore, MD, Nov. 8-11, 2005. 